\title{
Field-emission cathode gating for rf electron guns
}

\author{
J. W. Lewellen and J. Noonan \\ Advanced Photon Source, Argonne National Laboratory, 9700 S. Cass Avenue, Argonne, Illinois 60439, USA
}

(Received 23 December 2004; published 23 March 2005)

\begin{abstract}
We present a novel method of combining the most desirable characteristics of thermionic-cathode and photocathode rf guns, using a field-emission cathode and multiple rf frequencies. Simulations indicate that extremely low-emittance beams (on the order of $2 \mathrm{~nm}$ normalized emittance) at moderate beam currents $(1 \mathrm{~mA})$ and beam energies of $\sim 2 \mathrm{MeV}$ can be obtained using this technique. The resulting gun design promises to be useful as a driver source for a number of applications, including high-voltage electron microscopy, precision electron-beam welding, and long-wavelength $(\mathrm{THz})$ radiation generation; we include performance calculations for the electron microscopy and precision welding applications.
\end{abstract}

DOI: 10.1103/PhysRevSTAB.8.033502

PACS numbers: 41.75.Fr, 52.59.-f, 29.25.-t

\section{INTRODUCTION}

Most radio frequency (rf) electron guns constructed to date use either thermionic cathodes or photocathodes as their electron sources. Thermionic cathodes, which use high temperatures to induce electron emission from the cathode material, constantly emit electrons whenever the electric field in the gun is in the correct phase to accelerate electrons away from the cathode. Photocathodes use a light source, typically a high-power laser, to extract electrons from the photocathode.

Radio frequency electron guns work by establishing an oscillating electromagnetic field inside a cavity or series of cavities, with conducting walls. This field is used to accelerate electrons emitted from a cathode, down the bore of the gun, and out an exit port. Figure 1 is a schematic of a simple 1-cell rf gun. Electrons emitted from the cathode "early" in phase, between 0 and $\sim 60^{\circ}$ (region 1 in Fig. 2), will exit the gun with reasonable beam quality and comparatively high electron-beam energy, usually in the range of 2-5 MeV. Electrons emitted "too late" in phase cannot exit the gun before the electric field reverses sign (Fig. 2, regions 2 and 3); the beam will therefore be decelerated before it exits the gun. If emitted too late, electrons can reverse direction and strike the back wall of the cavity and the cathode, a process known as back bombardment [1]. Electrons emitted at the peak of the rf field (Fig. 2, region 2) will generally exit the gun, but with greatly degraded beam quality and lower beam energy.

Thermionic-cathode $\mathrm{rf}$ guns [2,3] can typically produce very high average power electron beams because of the continuous nature of the electron emission from the cathode, but can suffer from degraded beam quality because the electron emission cannot be gated to a particular fraction of an rf period. In addition, due to the requirements for high temperatures (ca. $1300^{\circ} \mathrm{C}$ ), thermionic cathodes are generally unsuitable for use in superconducting rf guns (which generally require operating temperatures $\leq 4 \mathrm{~K}$ ).

Photocathode $\mathrm{rf}$ guns $[4,5]$ can produce very highquality (bright) electron beams because the laser allows electron emission to be gated to a specific portion of the $\mathrm{rf}$ period, but most drive lasers cannot produce a laser pulse at every rf period. Therefore, for conventional normalconducting rf guns (e.g., built using copper cavities and operating at room temperature) the average beam power is typically lower than for a comparable thermionic-cathode rf gun. Further, photocathodes in common use typically offer a choice between either long lifetime and poor efficiency (thus requiring a far larger drive laser) or poor lifetime and high efficiency (requiring the use of a large cathode-fabrication and cathode-processing system adjacent to the electron gun) [6]. Superconducting rf photoinjectors, presently being designed for various applications, offer the promise of high average beam currents (ca. $1 \mathrm{~A}$ ) at high brightnesses (ca. $5 \mu \mathrm{m}$ normalized emittance) but will require very powerful cathode drive lasers; the cathode problem is not addressed simply by making the cavity superconducting.

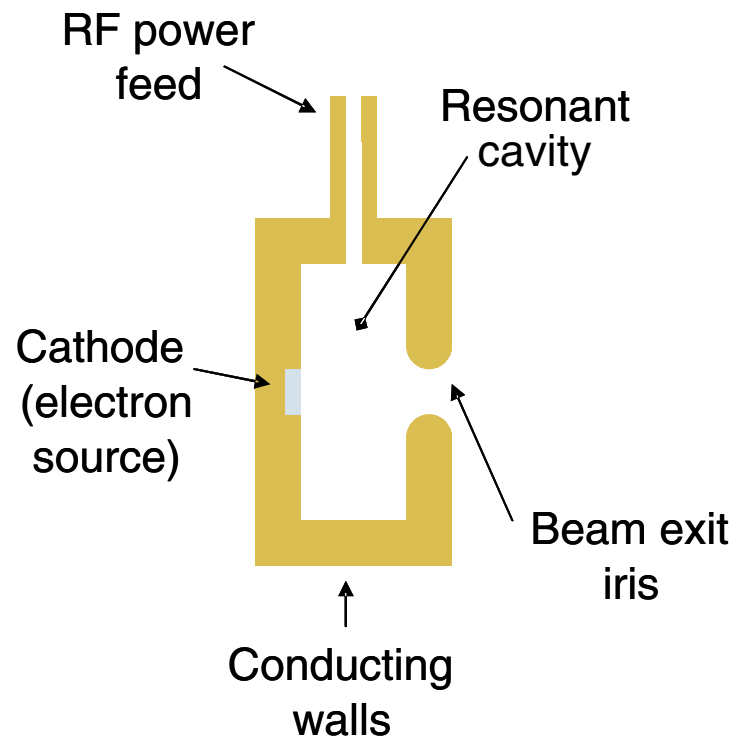

FIG. 1. (Color) Schematic of a single-cell rf electron gun. 


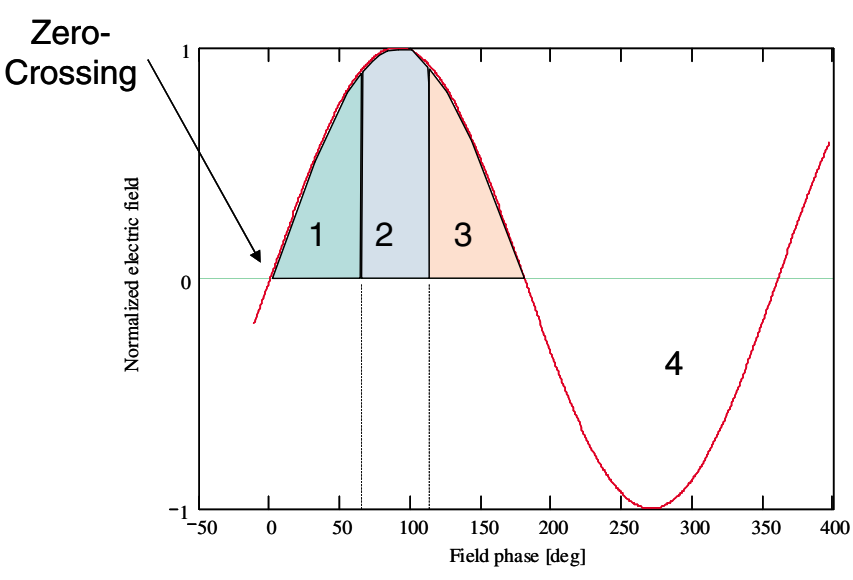

FIG. 2. (Color) Possible electron emission times.

Field-emission (FE) cathodes [7] operate by using strong electric fields to pull electrons from the cathode material directly. Thus, unlike thermionic cathodes, they do not emit continuously. Unlike photocathodes, their "triggering" mechanism does not rely on an external event such as the arrival of a laser pulse. Rather, FE cathodes do not emit electrons below a threshold electric field. Above that threshold, which can be varied significantly depending on the cathode design (and, in an rf cavity, the surface condition after rf conditioning), FE cathodes will begin to emit electrons, with the emission current increasing rapidly with increasing electric field [7]. At first glance, this behavior would seem to make FE cathodes a very appealing alternative to both thermionic and photocathodes. Difficulties arise because the FE cathode will emit the highest current when the electric field gradient is the strongest; the emission will thus be symmetric about the $90^{\circ}$ point. This is illustrated in Fig. 3. The resulting beam will typically have a very large energy spread, and very poor transverse quality. (This description applies directly to the "dark current" observed during the operation of some high-field rf photocathode guns. In these guns, imperfections on the photocathode surface act as FE cathodes. The resulting beams are typically low energy, with large energy spreads and exceedingly poor transverse beam quality [8].)

dc guns operate over a wide range of beam currents, from sub- $\mu \mathrm{A}$ electron-microscope guns to tens of $\mathrm{A}$ for electron-beam welders, and can operate with fieldemission, thermionic and photocathodes. They are typically limited in voltage to under $1 \mathrm{MV}$, however, with most applications running at less than $150 \mathrm{kV}$. The beam quality can be excellent, with nm-range emittances in electronmicroscope guns at low currents. dc-gun installations, particularly for high-power applications, typically require large dc power supplies and high-voltage systems.

This paper describes a general method for altering the emission time of a field-emitter cathode with respect to the rf phase in the gun. This approach combines the advantages

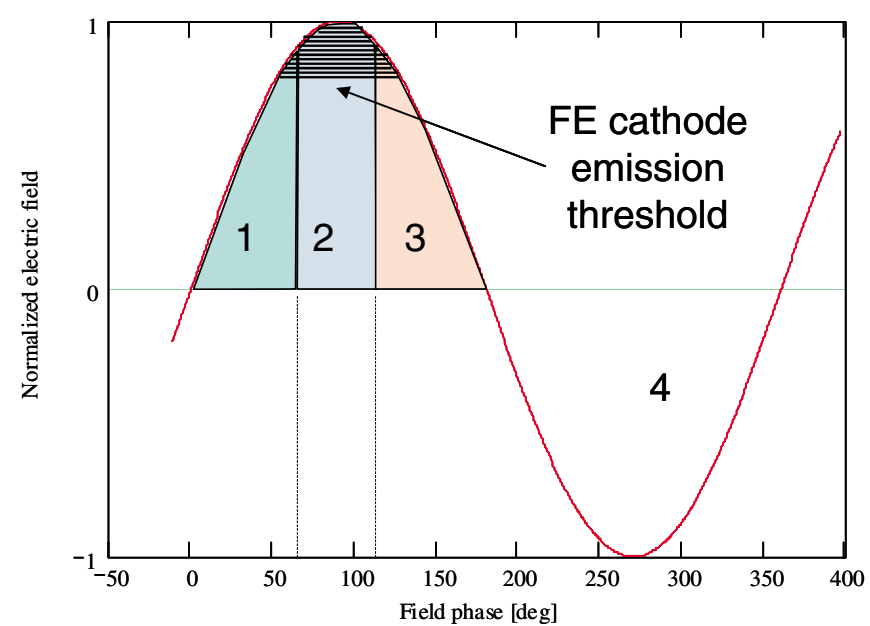

FIG. 3. (Color) FE cathode emission times during the rf period. The shaded region indicates the FE emission time.

of the thermionic-cathode rf gun (beam produced every rf period, no laser needed) with those of a photoinjector (gated emission at the most desirable time, highbrightness, superconducting rf compatible). The resulting design has an operating envelope different from both most common dc-gun designs and most rf-gun designs. It should have broad applicability across a number of application areas presently not available to conventional sources.

\section{RF FIELD ADDITION}

A given $\mathrm{rf}$ cavity is typically capable of supporting many different field patterns oscillating at many different frequencies; a specific pattern at a specific frequency is usually identified as a cavity mode [9]. Figure 4 shows the two lowest modes, or field patterns, for a single-cell rf gun. Typically, rf guns are designed to operate using a single mode in the cavity; often, but not always [10], the gun is designed to use the lowest frequency, or fundamental, mode. In this case, the two modes shown are the two lowest frequencies the cavity is capable of supporting; note that the higher frequency is not an exact harmonic (integer multiple) of the lower frequency.

It is possible to tune a cavity such that at least some of the modes are harmonic. For instance, it is possible to tune the cavity such that the third cavity mode oscillates at exactly 3 times the frequency of the fundamental mode. The fields in the cavity will then "beat" in phase with each other. (Some work has been performed using such a technique to generate an improved field for photoinjector $\mathrm{rf}$ guns [11-13].) Figure 5 illustrates the effect of adding or subtracting a 3rd-harmonic component to the fundamental, at a particular point in the rf cavity, as a function of the phase of the fundamental field.

At first glance, this does not appear to be particularly useful in that, although we can evidently control the duration of the peak field [expand into a flattop as in Fig. 5(a) or 

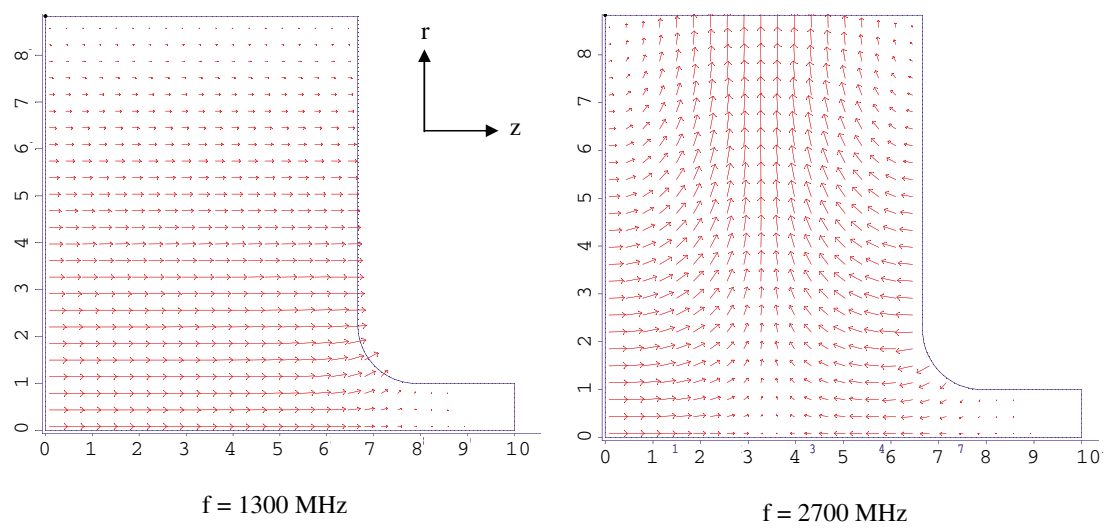

FIG. 4. (Color) Two modes for the same rf cavity. Plot axes are $r$ (radius) versus $z$ (axial) coordinates; all units are in centimeters. The arrows represent the direction and strength of the electric field in the cavity.

sharpen as in Fig. 5(b)], the peak field is still centered in region 2.

We can write the field addition as

$$
E_{\text {sum }}(t)=E_{1} \sin \left(\omega_{1} t+\phi_{1}\right)+E_{3} \sin \left(3 \omega_{1} t+\phi_{3}\right)
$$

where $\omega_{1}$ is the angular frequency of the fundamental field, $E_{1(3)}$ is the amplitude of the fundamental (3rd-harmonic) field, $\phi_{1(3)}$ is the phase of the fundamental (3rd-harmonic) field, and $t$ is time. We can choose to set $\phi_{1}=0$, and we can also write $E_{3}=\alpha E_{1}$, where $\alpha$ is simply a proportionality constant. In Fig. 5, in effect, $\alpha=1 / 9$, and $\phi_{3}=0^{\circ}$ for case (a) $180^{\circ}$ for case (b). Some linear accelerator designs include harmonic rf systems to help linearize the energy spread on the beam; they typically operate as in case (a) to remove the energy-versus-time curvature im- parted by accelerating a beam on the crest of a rf wave [11,13-15].

We may choose $\alpha$ and $\phi_{3}$ to be whatever we wish, however. In fact, should we choose $\alpha=0.4$ and $\phi_{3}=$ $-40^{\circ}$, we obtain the field shown in Fig. 6. Doing so results in two beneficial changes to the summed field. First, the width of the peak field has narrowed considerably, compared to the fundamental alone. Second, and most importantly, the peak of the field has shifted from $90^{\circ}$ to approximately $54^{\circ}$. Therefore, with an appropriately chosen emission threshold, the FE cathode will emit electrons around the $54^{\circ}$ point, within region 1 in Fig. 2. An example calculated emission profile, generated by inserting the rf electric field into the Fowler-Nordheim equation [16], is shown in Fig. 7. In this particular case the electric field is slightly too high; the second emission peak, at
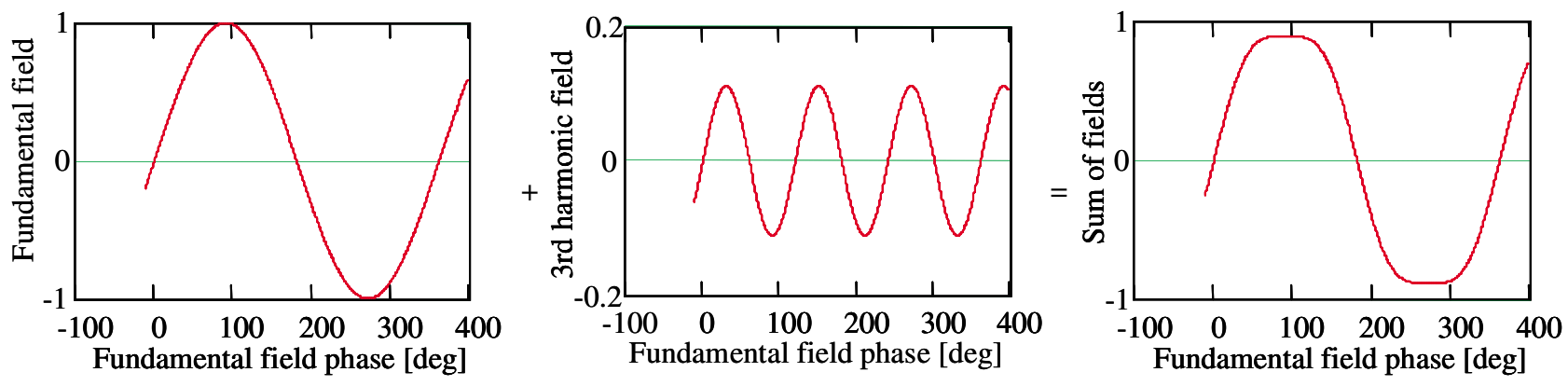

(a)
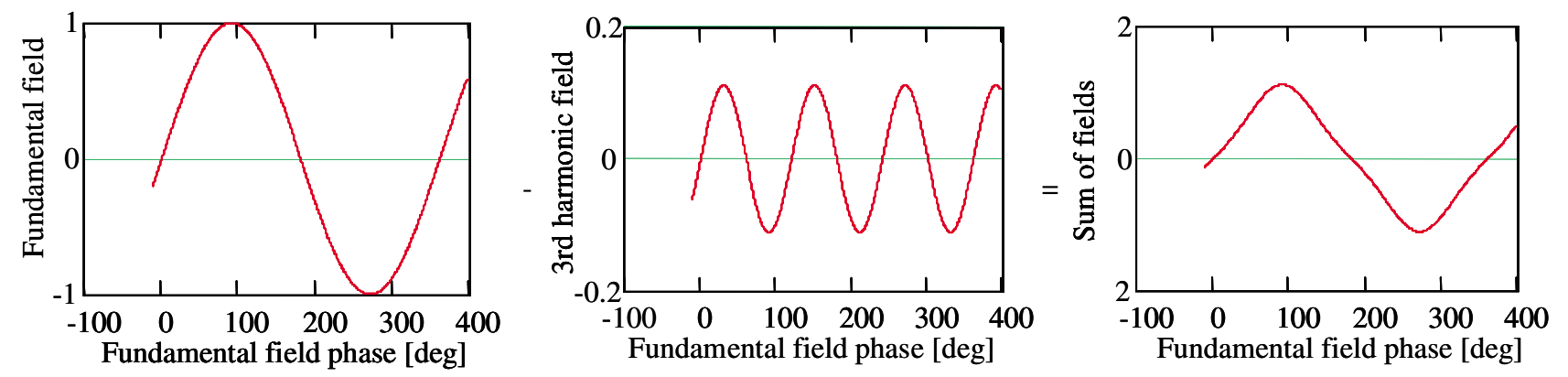

(b)

FIG. 5. (Color) Addition (a) and subtraction (b) of fundamental and 3rd-harmonic fields. 

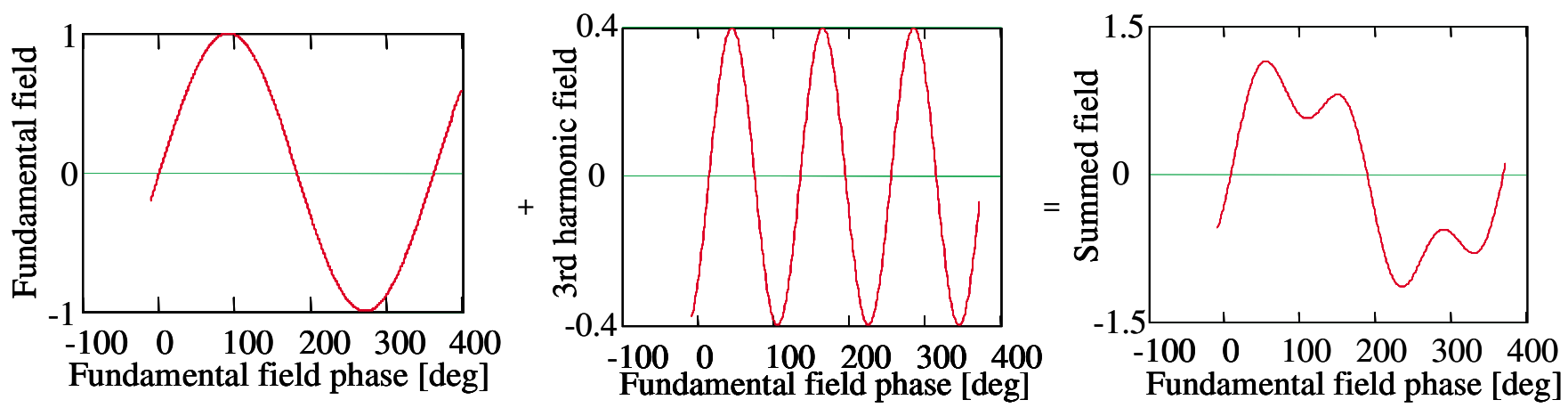

FIG. 6. (Color) Field sum of fundamental and 3rd-harmonic fields for $\alpha=0.4, \phi_{3}=-40^{\circ}$.

approximately $150^{\circ}$, is visible in the plot. Using a smaller electric field results in (1) the suppression of the second emission peak at $150^{\circ}$ and (2) both a narrowing and lowering of the emission peak at $54^{\circ}$. Therefore, by adjusting the phase and strength of the 3rd-harmonic field relative to the fundamental, we can cause a field-emission cathode to emit electrons at times optimized for the generation of high-brightness electron beams.

This summation of fields in the cavity represents, in effect, the first two terms of a Fourier series describing an ideal driving field for a field-emission cathode gun. In principle, additional improvements to the field shape could be made (e.g., generating a small flattop distribution) by adding more fields at higher harmonics. In practice, this rapidly becomes less practical due to both power-coupling and cavity resonance condition concerns.

We have also considered adding the 5th-harmonic component, rather than the $3 \mathrm{rd}$. This does not offer any obvious advantages in terms of beam quality and results in more "peaks" in the field sum. The result is that emission is not as cleanly gated to the desired time; instead, emission can occur at multiple times during the fundamental rf period, leading to the risk of "contaminating" the desired beam.

There are additional considerations that must be addressed before this technique can be applied to produce a viable electron-beam source. In particular, in order to obtain the properly gated electron emission as noted above, the 3rd-harmonic field has to be quite strong in comparison

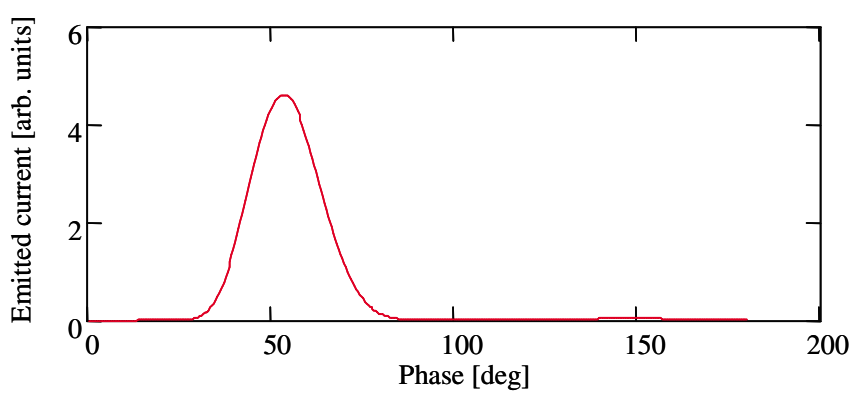

FIG. 7. (Color) Expected current emission profile, FE cathode, for the field shown in Fig. 6. to the fundamental field. For good beam dynamics in the gun, however, the fundamental field must dominate as the beam moves from the cathode to the exit. (The addition of a modest 3rd-harmonic field can benefit beam transport [13]; however, the required phase and amplitudes of the 3rd harmonic are those shown in Fig. 5(a), which are unsuitable for FE cathode gating.)

A method is therefore required to obtain a strong 3rdharmonic field component at the cathode, while minimizing its effects elsewhere in the cavity. This is accomplished as follows. The gun cavity contains a recess where the cathode would ordinarily be. The FE cathode is placed on a stalk located slightly behind the notional back wall of the cavity. The 3rd-harmonic field will penetrate into the recess more deeply than the fundamental, because of its higher frequency (and, therefore, shorter wavelength).

Thus, the 3rd-harmonic field will be strong, relative to the fundamental, at the cathode surface where it is required to properly gate the FE cathode emission. In the body of the gun, however, the fundamental will dominate, yielding dynamics similar to those of a conventional gun. The test geometry used for our simulations is shown in Fig. 8; the fundamental and 3rd-harmonic fields, plotted as distance along the axis of the gun, are shown in Fig. 9.

\section{CALCULATED PERFORMANCE}

The geometry shown in Fig. 8 was designed to meet the needs of initial performance calculations; in particular, the cavity radii and length were chosen to obtain a fundamental resonance at $1.3 \mathrm{GHz}$ ( $L$-band) and a 3rd-harmonic resonance at $3.9 \mathrm{GHz}$. The longitudinal position of the cathode stalk was chosen to obtain the desired field balance at the cathode tip and in the body of the cell, as described above and plotted in Fig. 9. The radii of the cathode stalk and cathode well were chosen to correspond to a coaxial line impedance of $50 \Omega$. The design has not otherwise been optimized for low emittance or other figures of merit.

The POISSON/SUPERFISH group of codes [17] was used to perform all cavity modeling; the simulation code PARMELA [18] version 3.35 was used to perform the majority of the beam dynamics calculations. The SDDS 


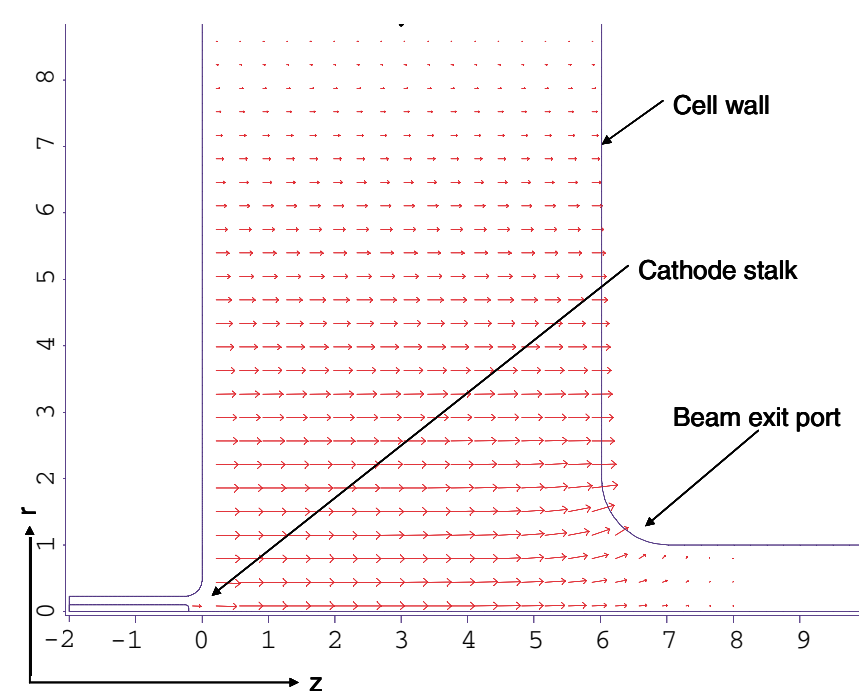

FIG. 8. (Color) FE cathode gun cell geometry. The fundamental cavity field is shown; dimensions are in centimeters.

ToolKit [19] was used for postprocessing; in particular, the SDDSANALYZEBEAM code was used to analyze the electronbeam parameters, rather than PARMELA's built-in calculation. (The results of the SDDSANALYZEBEAM calculation are in good agreement with PARMELA's built-in time-step calculation; however, data from SDDSANALYZEBEAM is in SDDS format, greatly easing other postprocessing tasks.)

A series of calculations were performed with a peak gradient of $20 \mathrm{MV} / \mathrm{m}$ on the cathode tip for the fundamental field and $8 \mathrm{MV} / \mathrm{m}$ on the tip for the 3rd harmonic. For each run a design average beam current was chosen; the current density was then varied by altering the radius of the emission area on the cathode surface. Figure 10 shows the normalized emittance $20 \mathrm{~cm}$ from the cathode tip,

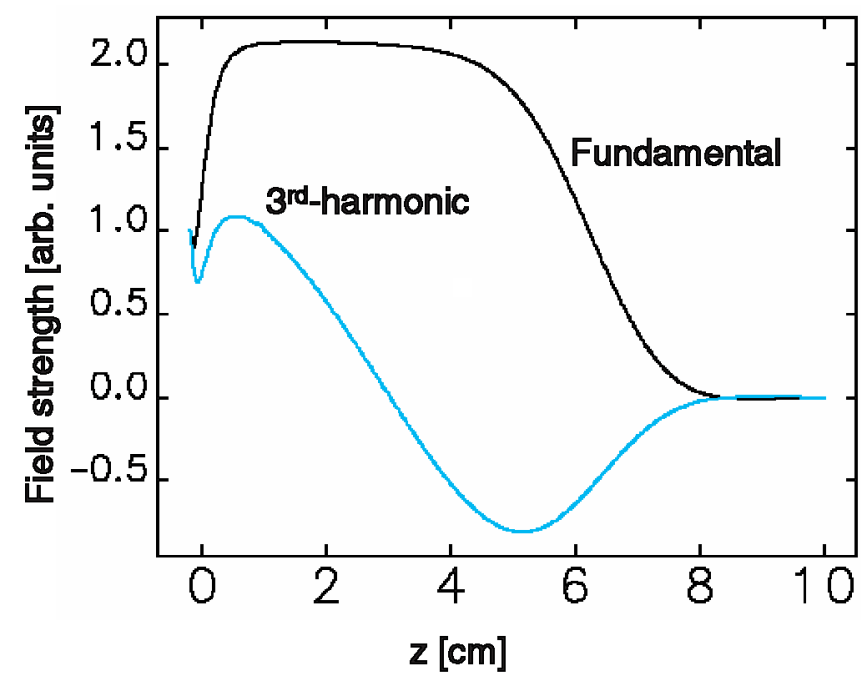

FIG. 9. (Color) Fundamental and 3rd-harmonic on-axis fields for the model gun cavity. The fields have been normalized to unity at the cathode surface.

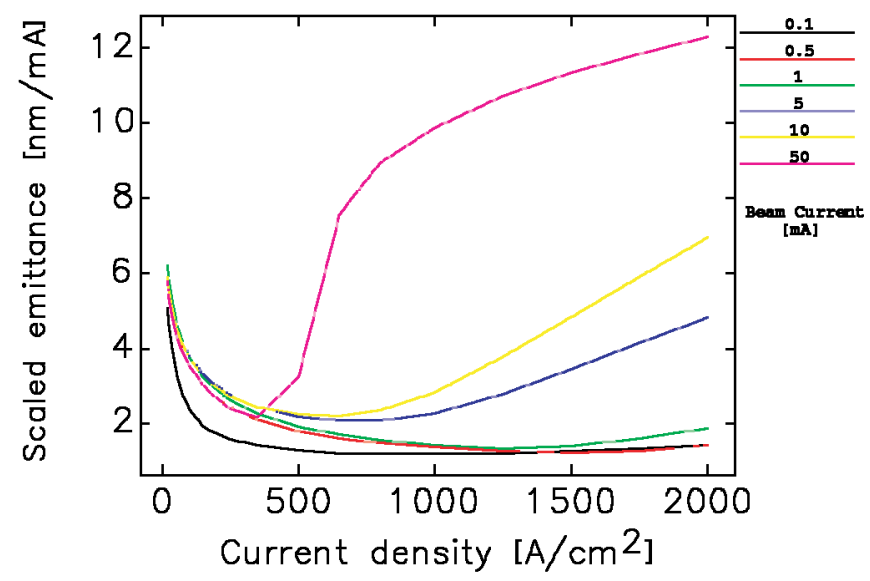

FIG. 10. (Color) Normalized scaled transverse emittance (e.g., normalized emittance divided by average beam current), as a function of time-average current density, for various beam currents.

divided by the beam current, for various beam currents and current densities. The vertical units are therefore $\mathrm{nm} / \mathrm{mA}$. (This scaling is done to allow a meaningful comparison of a wide range of beam currents on the same plot.) The beam emission area, or effective cathode radius, is calculated as

$$
R_{\text {cath }}=\sqrt{\frac{I_{\text {avg }}}{\pi j_{\text {avg }}}}
$$

where $R_{\text {cath }}$ is the (hard-edge) radius of the cathode, $I_{\text {avg }}$ is the time-average beam current, and $j_{\text {avg }}$ is the time-average current density.

At the highest beam current, $50 \mathrm{~mA}$, at higher current densities the space-charge forces become strong enough to begin seriously degrading the beam quality. This is also evident in Fig. 11, which shows the rms fractional energy spread as a function of current density for various average beam currents.

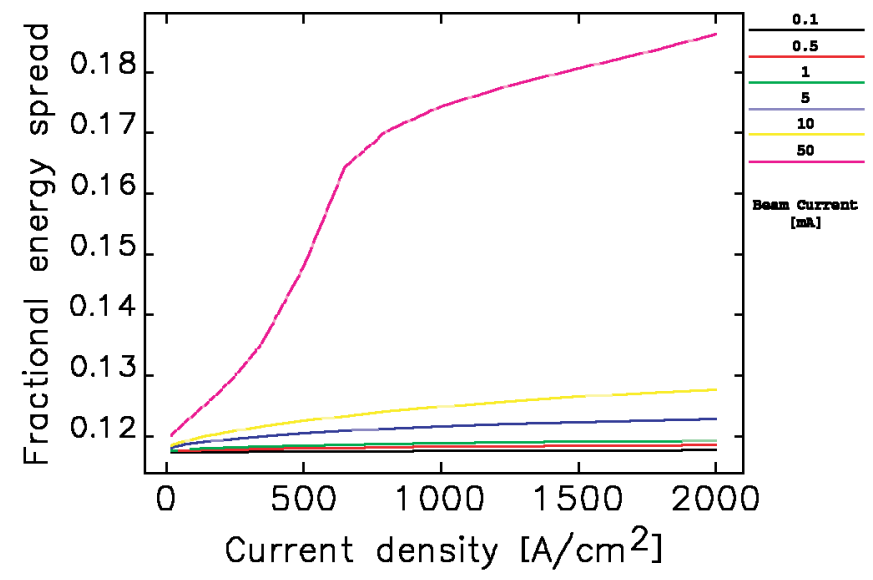

FIG. 11. (Color) rms fractional energy spread as a function of time-average current density, for various beam currents. 


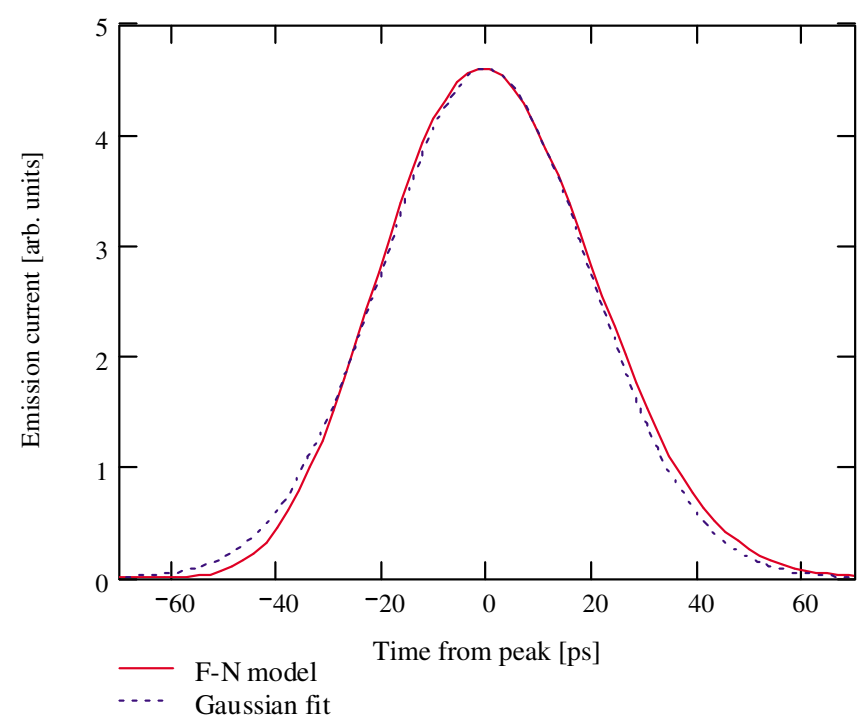

FIG. 12. (Color) Comparison of Fowler-Nordheim (F-N) calculated emission curve (solid red) with a fitted Gaussian (dotted blue). The F-N curve exhibits a slight asymmetry from the field addition.

The simulation results were checked with ASTRA [20] for the particular case of a 1-mA beam current. Because of differences in calculating the emittance, an energy-spread reduction cell was required for ASTRA to provide the same calculated emittance as PARMELA. In general, about a factor of 2 higher emittance was found with ASTRA than with PARMELA (2.3 nm from ASTRA versus $1.3 \mathrm{~nm}$ from PARMELA), with a cathode radius approximately $60 \%$ larger. While PARMELA uses a $z-r$ field map to determine the cavity fields, ASTRA calculates off-axis fields from the derivative of the on-axis field. Usually this is not an issue; in the particular case of the geometry used for this gun design, however, there are strong radial components of the field in close proximity to the cathode. This may account for the difference in results.

The first peak in Fig. 7 can be approximated reasonably well with a Gaussian curve, as shown in Fig. 12. A Gaussian longitudinal distribution was used in both PARMELA and ASTRA for convenience, as neither code has a built-in field-emission model ${ }^{1}$ suitable to generate the distribution directly. The particle-in-cell code SPIFFE [21] was modified with a Fowler-Nordheim emission model, and reproduced the expected distribution almost exactly.

It should be noted that these beams, especially at emission, have aspect ratios and other parameters considerably different from those of most high-brightness sources; we do not have a good benchmark of the codes' performance under these conditions. Unfortunately the SPIFFE code has a

\footnotetext{
${ }^{1}$ ASTRA does have a Schottky-enhancement model; however, this is for photoinjector applications rather than field-emission cathodes.
}

constant-size mesh; it is impractical to mesh the entire gun cavity at the resolution required to properly resolve the beam. Therefore, these results should be considered preliminary; however, they are sufficiently interesting as to warrant further investigation.

With the exception of SPIFFE, these simulations do not take into account the suppression of the cathode field in terms of the emitted current density; therefore, these results are expected to lose accuracy at high emission current densities. In general, these simulations were performed with the assumption that the gun is being operated at the maximum possible gradient before appreciable emission begins to occur from the secondary peak at $150^{\circ}$. The electron-beam bunch length and energy spread are at their maxima under these conditions. It is quite possible the beam quality would improve rapidly if the gradient were lowered, due to the smaller energy spread and shorter emission time. We hope to further explore this with a self-consistent emission model, perhaps with a custom field-emission cathode model added into GPT [22].

\section{CATHODE SELECTION AND SIMULATION}

Field-emission cathodes can be made of various materials, including silicon [23] and carbon nanotube [16,24]. The cathodes may be either gated, in which a grid placed close to the cathode is used to gate field emission, or ungated. For the application considered in this paper we assume ungated cathodes, with the effective gating being provided solely by the applied rf field.

The Fowler-Nordheim model describes the emission current density as a function of applied voltage, and, in simple form, can be expressed as [16]

$$
j=A \frac{\left(\beta E_{o}\right)^{2}}{\Phi} e^{-B\left(\Phi^{3 / 2} / \beta E_{o}\right)},
$$

where $j$ is the emission current density, $E_{o}$ is the "bulk," or mean, local field, $\beta$ is the field amplification factor due to the shape of the field emitter, $\Phi$ is the work function, and $A$ and $B$ are constants. (A more complex model is described in [25], and links to further considerations specific to superconducting cavities may be found at [26].) The work function typically has a value of $2-5 \mathrm{eV}$ [27]. The field enhancement factor $\beta$ is to an extent an adjustable parameter, for instance, depending on the diameter of the tubes as well as the geometry of the surface on which they are deposited. For this work we have chosen $\beta=7900$, within the range found in [16], and $\Phi=5 \mathrm{eV}$. (In actual operation the field-emitter parameters, $\beta$ in particular, can be altered by exposure to high rf fields, so there are potential lifetime issues beyond the scope of the present work.)

The intent of this work is not to determine conclusively the choice of an FE cathode material; carbon nanotubes offer some apparent advantages but are probably not the only possible choice. Rather, our primary goal is to explore 
the possibilities for, and performance of, a rf electron gun based around the use of an FE cathode.

\section{NOTES ON FREQUENCY SELECTION}

The examples above and the sample calculations below are based on the choice of a $1.3-\mathrm{GHz}$ fundamental $\mathrm{rf}$ frequency, with a corresponding 3rd harmonic at $3.9 \mathrm{GHz}$.

This particular choice of fundamental frequency was driven by three considerations. First, there are several commercial $\mathrm{rf}$ power sources available in the range needed for the $e$-microscope application considered below. Second, $L$-band cavities are a size that is a good compromise between machining tolerances (where lower frequencies are better) and compactness. Finally, the TESLA superconducting accelerator structures are designed to operate at $L$-band, so there is already a large and growing community knowledgeable about making superconducting cavities and associated systems in this frequency range. In short, it happens to be a convenient first choice.

There is nothing particularly magical about this selection of frequency, however, and arguments can be made for going to either lower or higher frequencies. It should be emphasized that the FE cathode gating method here will, in general, operate independently of the choice for the fundamental frequency. This is the addition of harmonic fields with a defined relationship in phase; therefore, everything scales with the fundamental frequency. This includes, for instance, the bunch length, which with lower (higher) frequency will become longer (shorter) in time, but which will have the same length when expressed in terms of degrees of rf phase. This has important implications for beam dynamics also, as it means that the basic performance should be maintainable across a broad range of frequency choices. The ability of the cavity to properly support and accelerate a given beam current does change somewhat with frequency, but in general is more limited by the available rf power than by the particular design of the cavity or choice of resonant frequency.

\section{SAMPLE APPLICATIONS}

We present two sample calculations using field-emission cathode gating and the gun design described above.

The initial goals for the first application were based on the needs of electron microscopy. Thus, emphasis was placed on reducing the beam emittance (i.e., improving transverse quality) and energy spread, while generating modest beam currents. For these simulations, the chosen bunch charge was $0.385 \mathrm{pC}$, or an average beam current of $0.5 \mathrm{~mA}$ if an electron bunch is produced every rf period.

\section{ELECTRON-BEAM MICROSCOPE}

PARMELA was used to simulate the beam line layout shown in Fig. 13. The energy filter introduces a correlation between the beam energy and position, allowing a narrow

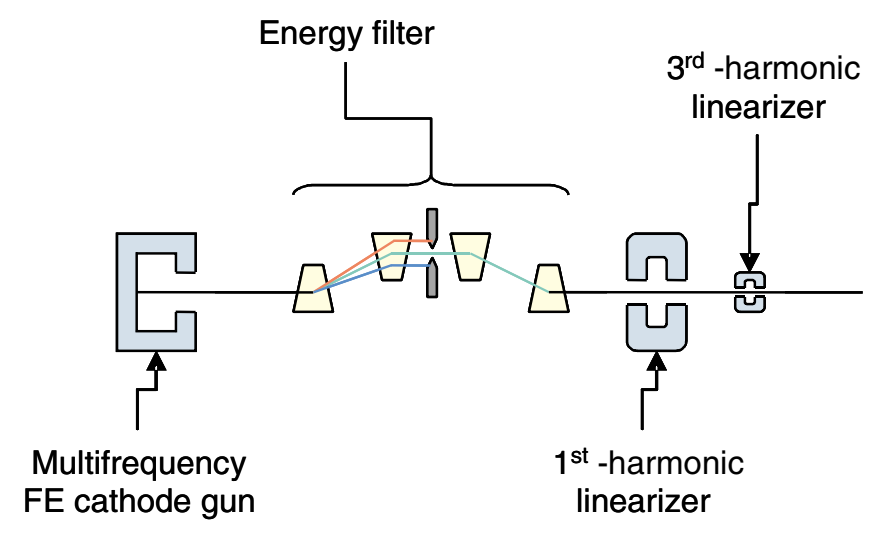

FIG. 13. (Color) Simulated beam line layout.

slice to be transmitted from the core of the beam. This results in both a reduced energy spread and an improved transverse quality, because the core of the beam generally is the portion where the transverse quality is highest.

A cathode $0.1 \mathrm{~mm}$ in diameter was assumed, generating an initial beam current of $0.5 \mathrm{~mA}$ on average. This is not at the optimum current density found on the curve shown in Fig. 10; it was chosen as a reasonable number for ease of fabrication. The applied fields were as those above, with a peak combined field on the cathode of about $25 \mathrm{MV} / \mathrm{m}$. The energy filter was set to transmit about $20 \%$ of the beam current. Finally, the 1st-harmonic linearizer reduces the beam energy spread by 2 orders of magnitude, and the 3rd-harmonic linearizer reduced the beam energy spread by another order of magnitude.

At the end of this simulated beam line, the beam current is about $90 \mu \mathrm{A}$. The average beam energy is $1.786 \mathrm{MV}$. The rms fractional energy spread is $1.7 \times 10^{-5}$, or about $30 \mathrm{~V}$ in absolute terms. The horizontal and vertical normalized emittances are $1.2 \times 10^{-3}$ and $1.0 \times 10^{-3} \mu \mathrm{m}$, respectively. (The difference arises because the energy filter bends the beam in the horizontal plane.) This should be sufficient to generate a beam spot about $1 \mathrm{~nm}$ in radius, given good electron-beam optics. The total electron-beam power is about $180 \mathrm{~W}$. (The beam power from the gun is closer to $900 \mathrm{~W}$; the scrapers in the energy filter absorb the difference.) Therefore, the power density on the spot could in principle be approximately $57 \mathrm{GW}$ per square millimeter. Reducing the transmission of the filter further will result in additional improvements to beam quality at the expense of current.

\section{ELECTRON-BEAM WELDER}

If the energy filter is removed from the beam line, thereby passing all of the beam current, the 1st- and 3rdharmonic linearizers can still be used to reduce the beam energy spread. In this case, the beam energy is around $1.4 \mathrm{MV}$ and the final energy spread is $1.7 \times 10^{-4} \mathrm{rms}$ (or about $300 \mathrm{~V}$ ). Figure 14 shows longitudinal phase-space plots (a) at the exit of the gun, (b) after the fundamental 
(a)

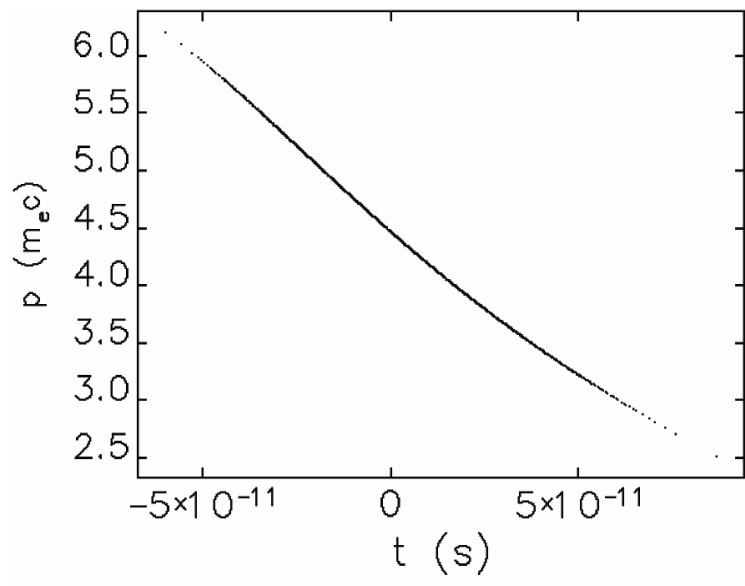

(b)

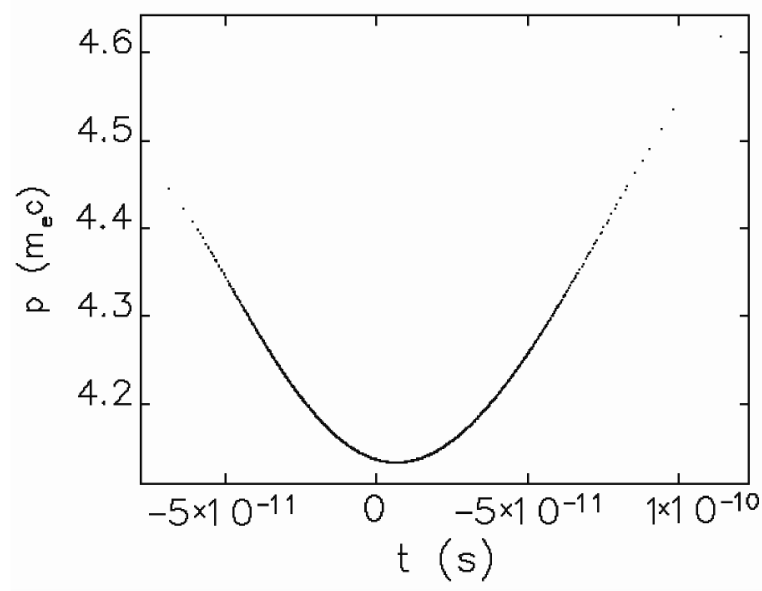

(c)

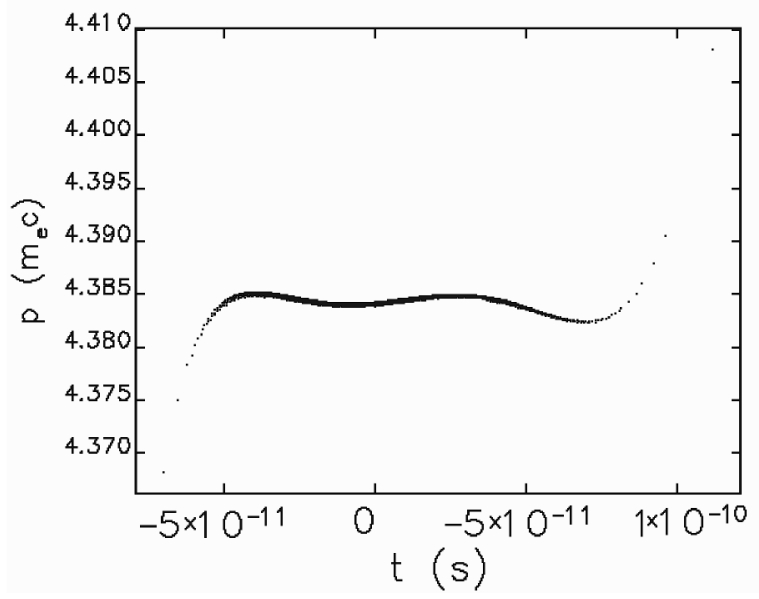

FIG. 14. Longitudinal phase space in "welder" configuration, (a) after gun, (b) after 1st-harmonic linearizer, and (c) after 3rdharmonic linearizer.

linearizer cavity, and (c) after the 3rd-harmonic linearizer cavity. The beam energy is lower than above, and the energy spread is larger, because the energy filter is not removing the "wings." Thus, a different minima for the energy spread is found. The transverse quality is also worse, at about $4 \times 10^{-3} \mu \mathrm{m}$. On the other hand, the entire beam current of $0.5 \mathrm{~mA}$ is transmitted, for a final beam power of about $700 \mathrm{~W}$.
As a comparison, a typical electron-beam welder might have a beam power of $15 \mathrm{~kW}$, with a voltage of $60 \mathrm{kV}$. Thus, although the beam power is higher, the $e$-beam welder's beam energy is lower by a factor of 20 . The beam from the multifrequency gun should therefore penetrate more deeply into the material, and should almost certainly be able to provide higher-precision, smallerarea welds.

It is also notable that the beam power, $700 \mathrm{~W}$, can easily be provided for by relatively compact, $\mathrm{cw}$ rf power sources. This would result in an $e$-beam welder that is smaller and more compact, because high-voltage high-current dc power supplies would no longer be needed.

If the cathode radius were to be doubled, to $0.2 \mathrm{~mm}$, and the beam current increased by an order of magnitude, to $5 \mathrm{~mA}$, the final energy spread remains approximately the same at $1.8 \times 10^{-4}$, and the emittance increases to $2.6 \times$ $10^{-2} \mu \mathrm{m}$, roughly in proportion to the electron-beam current. The beam power increases to $7 \mathrm{~kW}$.

\section{PENETRATION DEPTHS}

The penetration of an electron beam into matter scales (at low energies) approximately as

$$
\delta z \approx 0.1 E^{1.5} / \rho,
$$

where $\delta z$ is the penetration depth in $\mu \mathrm{m}, E$ is the beam energy in $\mathrm{kV}$, and $\rho$ is the material density in $\mathrm{g} / \mathrm{cm}^{3}$. (This is an empirical formula, but is in reasonable agreement with theoretical calculations.) For instance, a $15-\mathrm{kV}$ electron beam should penetrate about $2.3 \mu \mathrm{m}$ into a silicate material with a density of $2.5 \mathrm{~g} / \mathrm{cm}^{3}$.

Given a notional $100-\mathrm{kV}$ beam energy for an electron microscope, the beam from the FE cathode gun, configured to run with the energy filter and a final beam energy of $1.7 \mathrm{MeV}$, could be expected to penetrate approximately 70 times as deeply into a sample, all other things being equal.

For a typical electron-beam welder operating at $60 \mathrm{kV}$, the expected penetration depth into iron or copper would be around $5.5 \mu \mathrm{m}$. (Actual welds can go much deeper due to heat diffusion, etc.) The beam from the FE cathode gun without the energy filter, with a final beam energy of $1.4 \mathrm{MeV}$, should penetrate $0.6 \mathrm{~mm}$, more than 100 times as deep, and therefore deposit more of the electron-beam energy into the volume of the metal as opposed to on the surface.

\section{DISCUSSION AND CONCLUSIONS}

We have presented a method for gating the emission from a field-emission cathode in such a fashion as to make the FE cathode a viable choice for high-brightness $\mathrm{rf}$ electron gun design. The resulting design functions in a portion of parameter space generally unoccupied by either 
existing dc-gun or rf-gun sources. Performance figures were calculated for applications to electron microscopy and electron-beam welding.

If superconducting cavities are used for the gun and linearizer cavities, there is effectively no power lost in the cavity walls, and the rf power system can consist of relatively low-power, compact oscillator sources. This would maintain a relatively compact footprint for an electron-microscope device and should potentially reduce the footprint for an electron-beam welder. Potential advantages over existing electron-microscope designs include greater beam energy and resulting penetration depth, and the pulsed nature of the beam. Potential advantages over existing electron-beam welder designs include the potentially compact nature of the source and power supply, highpower density, and increased penetration depth.

Other applications of interest include the use of the gun and linearizer to provide beam for a compact free-electron laser operating in the $\mathrm{THz}$ region.

\section{ACKNOWLEDGMENTS}

The authors wish to thank Courtlandt Bohn (Northern Illinois University), David Dowell (Stanford Linear Accelerator Center), and Todd Smith (Stanford University) for interesting discussions, support, and encouragement. This work was supported by the U.S. Department of Energy, Office of Basic Energy Sciences, under Contract No. W-31-109-ENG-38.

[1] T. Yamaguchi et al., in Proceedings of the 22nd International Free-Electron Laser Conference, Durham, NC, 2000 (Elsevier, Amsterdam, 2001), pp. 588-592.

[2] J. W. Lewellen et al., in Proceedings of the 1998 Linear Accelerator Conference, Chicago, IL (IEEE, Piscataway, NJ, 1998), pp. 863-865.

[3] S. Park, in Proceedings of the 2002 Linear Accelerator Conference, Gyeongju, Korea (Pohang Accelerator Laboratory, Pohang, Korea, 2002), pp. 322-324.

[4] J.E. Clendenin, in Proceedings of the 1996 Linear Accelerator Conference, Geneva, Switzerland (CERN, Geneva, 1999), pp. 298-302.

[5] C. Travier, Nucl. Instrum. Methods Phys. Res., Sect. A 340, 26 (1994).

[6] D. H. Dowell, in Free-Electron Laser Challenges II, SPIE Proceedings Vol. 3614 (SPIE-International Society for Optical Engineering, Bellingham, WA, 1999), pp. 14-21.
[7] Y. Cheng and O. Zhou, C.R. Physique 4, 1021 (2003).

[8] John Schmerge (private communication).

[9] J.D. Jackson, Classical Electrodynamics (Wiley, New York, 1975), 2nd ed.

[10] J. W. Lewellen, Phys. Rev. ST Accel. Beams 4, 040101 (2001).

[11] T. I. Smith, in Proceedings of the 1986 Linear Accelerator Conference, Stanford, CA (SLAC Report No. SLAC-R303, 1986), pp. 421-426.

[12] L. Serafini, in Advanced Accelerator Concepts, edited by J. S. Wurtele, AIP Conf. Proc. No. 279 (AIP, New York, 1993), pp. 645-674.

[13] D. H. Dowell et al., Nucl. Instrum. Methods Phys. Res., Sect. A 528, 316 (2004).

[14] P. Emma, SLAC Technical Note No. LCLS-TN-01-1, 2001.

[15] T. Zwart et al., in Proceedings of the 2003 Particle Accelerator Conference, Portland, OR (IEEE, Piscataway, NJ, 2003), pp. 980-982.

[16] D. Y. Zhong et al., Appl. Phys. Lett. 88, 506 (2002).

[17] J.H. Billen and L.M. Young, Los Alamos National Laboratory Report No. LA-UR-96-1834, 2003.

[18] L. M. Young, Los Alamos National Laboratory Report No. LA-UR-96-1835, 2004.

[19] The SDDS ToolKit was initially conceived and implemented by M. Borland; it is available online at http:// www.aps.anl.gov/asd/oag/software.shtml

[20] K. Flöttmann, computer code ASTRA, http://www.desy.de/ $\sim$ mpyflo/ (executable code and users manuals are available online).

[21] M. Borland, computer code SPIFFE, http://www.aps.anl.gov/asd/oag/software.shtml

[22] Pulsar Physics, commercial code GPT, http://www. pulsar.nl/

[23] D. Temple et al., in Proceedings of the IEEE Vacuum Microelectronics Conference, Portland, OR, 1995 (IEEE, Piscataway, NJ, 1995), pp. 113-117.

[24] H. Manohara et al., in Micromachining and Microfabrication Conference, SPIE Proceedings Vol. 5343 (SPIE-International Society for Optical Engineering, Bellingham, WA, 2004), pp. 227-234.

[25] Alex Chou, Handbook of Accelerator Physics and Engineering (World Scientific, Singapore, 1999).

[26] Part of a general primer on superconducting rf cavities, this page is the start of a discussion of Fowler-Nordheim theory in relation to various srf cavity concerns: http:// www.lns.cornell.edu/public/CESR/SRF/BasicSRF/ SRFBas31.html

[27] T. C. Choy, A. H. Harker, and A. M. Stoneham, J. Phys. Condens. Matter 16, 861 (2004). 